\title{
Commentary on: Absence of Stimulus-driven Synchronization Effects on Sensory Perception in Autism: Evidence for Local Under- Connectivity
}

Mark Tommerdahl

Department of Biomedical Engineering, University of North Carolina, USA

Article Info

\section{Article Notes}

Received: 04/08/2016

Accepted: 05/03/2016

\section{Correspondence:}

Dr. Mark Tommerdahl

Department of Biomedical Engineering

University of North Carolina

Chapel Hill, NC 27599, USA

Email: mark_tommerdahl@med.unc.edu

C 2016 Tommerdahl M. This article is distributed under the terms of the Creative Commons Attribution 4.0 International License
Autism is a pervasive developmental disorder that affects many aspects of the central nervous system, and a number of the deficits manifested in the disorder could be attributed to disruptions in functional connectivity. These disruptions - which could occur both locally as well as globally - motivated us to develop metrics that would be sensitive to alterations in cortical-cortical interactions. In 2007 , we reported a method for perceptually assessing the impact of stimulus-driven synchronization of cortical ensembles that showed significant promise for perceptually measuring local connectivity ${ }^{1}$. In that report, we demonstrated that delivering relatively weak sinusoidal stimuli to tips of digits 2 and 3 (D2 and D3) had a significant impact on an individual's temporal order judgement (TOJ). The use of a number of other stimulus conditions presented simultaneously during the TOJ task - which did not have a significant impact on the individual's TOJ - led us to develop a theory that synchronization of cortical ensembles in primary somatosensory cortex (SI) could significantly impact the topography of temporal perception. Additionally, we proposed that the impact that such synchronizing conditioning stimuli have on TOJ - which can be measured relatively quickly - could provide an effective means to assess the functional connectivity of neurologically compromised subject populations.

In a subsequent paper ("Absence of stimulus-driven synchronization effects on sensory perception in autism: Evidence for local under-connectivity?"), we utilized the method to assess the functional connectivity of adult individuals with autism. Since a number of neurophysiological characteristics demonstrated in autism share the common theme of under-connectivity in the cerebral cortex, and one of the prominent theories of the cause of the dysfunctional connectivity in autism is based on distinct anatomical structures that differ between the autistic and the neurotypical cortex, we hypothesized that stimulus-driven synchronization of cortical ensembles would have little or no impact on TOJ in individuals with autism. Our reasoning centered on the differences in minicolumnar structure that have been identified between the autistic and typically developing brain ${ }^{2}$. The functional minicolumn has been identified as occupying a much smaller space in the cortex of individuals with autism as compared to neurotypical controls, and this aberration in architecture has been proposed to reflect under-connectivity at the local or within-macrocolumn level. Furthermore, it was proposed that the under-connectivity within the macrocolumn subsequently leads to the dysfunctional connectivity 
that is observed globally across cortical areas in autism Numerous reports have indicated reduced functional connectivity on a large scale in the brains of individuals with autism, and we hypothesized that if the larger-scale aberrant dynamics in autism were due - at least in part to a widespread propagation of the errors introduced at the level of local connectivity between minicolumns, then aberrations in local functional connectivity should also be detectable. This was, in fact, what we demonstrated in our report - that local functional connectivity, as measured via TOJ observations in the presence and absence of synchronized conditioning stimuli - was much lower than that observed in typically developing controls.

The paper provides evidence that local functional connectivity in autism is weak, and it speculates on two things that cannot currently be proven: (1) that the underlying cause of that lower than normal connectivity is related to the alterations in minicolumnar structure that Manuel Casanova and colleagues have provided anatomical evidence for, and (2) that the widespread reports of lower than normal global connectivity could be accounted for by lower than normal local connectivity. However, there have been a number of subsequent reports that are consistent with aberrant local connectivity in autism and a number of reports that provide further support for the measure that we described as being reflective of local under-connectivity.

\section{Observations Consistent with the Original Findings}

Probably the most significant report relevant to our autism local under-connectivity findings, to date, is the work by Tal Kenet and colleagues that demonstrates under-connectivity in autism ${ }^{3}$. Their MEG observations demonstrated under-connectivity at the local level as a result of delivering simultaneous synchronized $25 \mathrm{~Hz}$ stimuli to digits 2 and 3, essentially the same conditions that were used to deliver stimuli in our first report. Additionally, this group was also able to demonstrate differences in global feedforward and feedback inhibition between autism and typically developing populations that does not contradict the idea that local under-connectivity could play a role in global under-connectivity in autism. Several other neurophysiological studies lend support to the idea of local under-connectivity in autism. Studies utilizing diffusion tensor imaging (DTI) ${ }^{4}$, magnetoencephalography (MEG) ${ }^{5}$, and electroencephalography (EEG) ${ }^{6-7}$ have all yielded results that were consistent with our original finding. Additionally, some behavioral studies ${ }^{8-9}$ and examination of processing in other sensory systems ${ }^{10-13}$ also support decreased local functional connectivity in autism spectrum disorder (ASD).

\section{Observations inconsistent with the Original Findings}

Nick Puts and colleagues ${ }^{14}$ demonstrated no significant difference between autistic and typically developing control
(TDC) individuals with the local functional connectivity measure, but their observations were obtained from adolescents, whereas the study that we originally conducted was restricted to adults. In fact, there were even significant differences in simple TOJ - in the absence of conditioning stimulation - observed in the adults in our report and the adolescents in the Puts report. Adolescents were much worse at the TOJ performance task and had much higher scores. The local functional connectivity metric relies on observations of TOJ in the presence and absence of synchronized conditioning stimuli, and the significant difference in the TOJ task could at least partially explain the differences observed between adults and adolescents.

\section{What could account for disruptions in local functional connectivity?}

As one might suspect, local functional connectivity can be impacted for a number of reasons - not just the minicolumnar-based reason that we originally proposed for being responsible for the difference observed in individuals with autism versus typically developing individuals. Since the publication of our first report, it has been demonstrated that this metric of local functional connectivity can be impacted by a number of conditions. For example, Aimee Nelson and colleagues showed that suppression of SI cortical activity via theta burst stimulation reduces the impact of stimulus-driven synchronization on TOJ task performance in healthy controls ${ }^{15}$. In other words, blocking neural activity in primary somatosensory cortex leads to a degradation of the TOJ-based functional connectivity metric.

Neurological disorders other than autism have been demonstrated to lead to disruptions in local functional connectivity. Individuals with migraine demonstrate local under-connectivity ${ }^{16}$ as do individuals with concussion ${ }^{17}$. However, the reason for the disruption in these two populations is most likely the result of different alterations. Pain - such as the pain induced by migraine - promotes activity in cortical area 3a, which consequently results in the disruption of synchronized activity due to interactions between area $3 \mathrm{a}$ and areas $3 \mathrm{~b}$ and $1^{18-19}$. The mechanism that impacts local functional connectivity in concussion is purely speculative at this point, but it is doubtful that area $3 \mathrm{a}$ is involved in the manner that it is with migraine pain, and experiments are currently underway that target the mechanisms involved in concussion. With noxious stimulation, we have found that stimulus conditions both in the presence and absence of noxious heat - can significantly impact the stimulus-driven synchronization of SI cortical neurons ${ }^{19}$. The overriding theme is that local functional connectivity is dependent on a number of mechanisms being intact, and while the underlying minicolumnar pathology undoubtedly plays a significant role in that functional connectivity ${ }^{20}$, neurophysiological disruptions can also have an impact on it. 


\section{Experimental Evidence Provides Support for the TOJ-based Metric as Being Reflective of Local Functional Connectivity.}

Our initial hypothesis was that the topography of temporal perception was altered with synchronized stimulus driven activity. In order to test this idea, we made observations with extracellular recordings from D2 and D3 cortical representations in SI of non-human primates under various stimulus conditions. When a simple pulsatile stimulus was delivered to D2, there was, as expected, pronounced stimulusevoked activity in the D2 cortical representation and a very weak stimulus-evoked response in the D3 representation. When that same pulsatile stimulus was delivered to D2 in the presence of synchronized conditioning stimulation of D2 and D3 (emulating the stimulus conditions of the TOJ functional connectivity task), there was a significant stimulus evoked response not only in the D2 representation, but also in the D3 representation ${ }^{21}$. In other words, the conditioning stimulus functionally linked the two digits by allowing crosstalk between the two digit representations. Thus, the linkage between the digit representations engages dynamically as a function of the stimulus-driven activity delivered to the adjacent cortical ensembles and demonstrates a cortical correlate of the perceptual measures that we obtained in the typically developing population. Obviously, the shortcoming of this work is the absence of an animal model of autism; such a model would allow us to complete the loop between the neurophysiological basis of the functional connectivity measure and the alteration that we observed that predicts local under-connectivity in the autism population.

\section{Local vs. Global Functional Connectivity Differences in Autism}

For quite some time, autism has been characterized by many in the field as having global under connectivity while at the same time demonstrating local over connectivity. The characterization of local under-connectivity was based on observations that many individuals with autism are hyper-sensitive to environmental stimuli and have lower than normal thresholds of stimulus detection. However, it should be pointed out that lower than normal inhibition will lead to hyper-excitation; this should not be confused with hyper-connectivity. In fact, lower than normal inhibition is most likely a reflection of lower than normal functional connectivity. Although it cannot be proven directly, we still support our original idea that local functional underconnectivity plays a role in the information processing deficits observed in autism. This is consistent with results obtained from ongoing computational modeling work ${ }^{22}$ that perhaps, coupled with better research techniques in the future will point to ways to not only detect, but alter, the local functional connectivity that may be playing a significant role in the disruptions in global connectivity and consequently, information processing deficits in autism.

\section{References}

1. Tommerdahl M, Tannan V, Zachek M, Holden JK, Favorov OV. Effects of stimulus-driven synchronization on sensory perception. Behav Brain Funct. 2007; 3(1): 1

2. Casanova MF, van Kooten IAJ, Switala AE, van Engeland $H$, Heinsen $H$, Steinbusch HWM, Hof PR, Trippe J, Stone J, Schmitz C. Minicolumnar abnormalities in autism. Acta Neuropathol. 2006; 112: 287-303.

3. Khan S, Michmizos K, Tommerdahl M, Ganesan S, Kitzbichler MG, Zetino $\mathrm{M}$, et al. Somatosensory cortex functional connectivity abnormalities in autism show opposite trends, depending on direction and spatial scale. Brain. 2015; 138: 1394-1409.

4. Shukla DK, Keehn B, Smylie DM, Müller RA. Microstructural abnormalities of short-distance white matter tracts in autism spectrum disorder. Neuropsychologia. 2011; 49(5): 1378-1382.

5. Coskun MA, Loveland KA, Pearson DA, Papanicolaou AC, Sheth BR Functional assays of local connectivity in the somatosensory cortex of individuals with autism. Autism Res. 2013; 6(3): 190-200.

6. Mathewson KJ, Jetha MK, Drmic IE, Bryson SE, Goldberg JO, Schmidt LA. Regional EEG alpha power, coherence, and behavioral symptomatology in autism spectrum disorder. Clin Neurophysiol. 2012; 123(9): 17981809.

7. Jamal W, Das S, Oprescu IA, Maharatna K, Apicella F, Sicca F. Classification of autism spectrum disorder using supervised learning of brain connectivity measures extracted from synchrostates. J Neural Eng. 2014; 11(4): 046019.

8. Baranek GT, Watson LR, Boyd BA, Poe MD, David FJ, McGuire L. Hyporesponsiveness to social and nonsocial sensory stimuli in children with autism, children with developmental delays, and typically developing children. Dev Psychopathol. 2013; 25(2): 307-320.

9. Boyd BA, Baranek GT, Sideris J, Poe MD, Watson LR, Patten E, et al. Sensory features and repetitive behaviors in children with autism and developmental delays. Autism Res. 2010; 3(2): 78-87.

10. Haigh SM, Minshew N, Heeger DJ, Dinstein I, Behrmann M. Overresponsiveness and greater variability in roughness perception in autism. Autism Res. 2016. 9(3): 393-402

11. Kéïta L, Mottron L, Bertone A. Far visual acuity is unremarkable in autism: do we need to focus on crowding? Autism Res. 2010; 3(6): 333-341.

12. Riquelme I, Hatem SM, Montoya P. Abnormal pressure pain, touch sensitivity, proprioception, and manual dexterity in children with autism spectrum disorders. Neural Plasticity. 2016; 1723401.

13.Wada M, Suzuki M, Takaki A, Miyao M, Spence C, Kansaku K. Spatiotemporal processing of tactile stimuli in autistic children. Sci Rep. 2014; 4: 5985.

14.Puts N, Wodka E, Tommerdahl M, Mostofsky S, Edden R. Impaired tactile processing in children with autism spectrum disorder. J Neurophysiol. 2014; 111: 1803-1811.

15.Lee J, Favorov OV, Tommerdahl M, Lee J, Whitsel B. Attenuated glial $\mathrm{K}+$ clearance contributes to long-term synaptic potentiation via depolarizing GABA in dorsal horn neurons of rat spinal cord. Exp Neurobiol. 2014; 23(1): 53-64.

16. Nguyen R, Ford S, Calhoun A, Holden J, Gracely R, Tommerdahl M. Neurosensory assessments of migraine. Brain Res. 2013; 1498: 50-58.

17. Tommerdahl M, Dennis RG, Francisco EM, Holden JK, Nguyen R, Favorov OV. Neurosensory assessments of concussion. J Mil Medicine, In Press; 2016.

18.Vierck C, Whitsel BL, Favorov OV, Brown A, Tommerdahl M. Role of primary somatosensory cortex in the coding of pain. Pain. 2013; 154: 334-344. 
19. Whitsel B, Favorov O, LiY, Lee J, Quibrera M, Tommerdahl M. Nociceptive afferent activity alters the SI RA neuron response to mechanical skin stimulation. Cereb Cortex. 2010; 20(12): 2900-2915.

20. Favorov OV, Whitsel BL, Tommerdahl M. Discrete, place-defined macrocolumns in aomatosensory cortex: Lessons for modular organization of the cerebral cortex. Recent Advances on the Modular Organization of the Cortex. 2015; 143-179.
21. Francisco E, Favorov OV, Tommerdahl M. The role of cortical modularity in tactile information processing: An approach to measuring information processing deficits in autism. Recent Advances in Autism Spectrum Disorder-Volume II. 2013; 1147

22. Favorov OV, Awan O, Tommerdahl M. Minicolumnar model of somatosensory perceptual abnormalities in autism. Society for Neuroscience $346.5 ; 2008$. 